\title{
Effects of Copolymer Composition on the Crystallization and Morphology of Poly(E-caprolactone)-block-Polystyrene
}

\author{
Shuichi Nojima, ${ }^{\dagger}$ Masato Fujimoto, Hiroshi KaKinira, \\ and Shintaro SASAKI
}

School of Materials Science, Japan Advanced Institute of Science and Technology

(JAIST), Tatsunokuchi, Ishikawa 923-1292, Japan

(Received May 12, 1998)

\begin{abstract}
The morphology and melting behavior of poly( $\varepsilon$-caprolactone)-block-polystyrene (PCL- $b$-PS) copolymers, quenched from the melt or cast from the toluene solution, were investigated by small-angle X-ray scattering (SAXS) and differential scanning calorimetry (DSC) as a function of copolymer composition. The glass transition temperature of PS blocks is higher than the melting temperature of PCL blocks, $T_{\mathrm{m} . \mathrm{PCL}}$, in this system, so that the molecular motion is extremely restricted when the PCL block crystallizes in temperatures below $T_{\mathrm{m}, \mathrm{PCL}}$. DSC results showed that the quenched PCL- $b$-PS did not crystallize at any temperature when PCL vol $\%, \phi_{\mathrm{PCL}}$, was less than $26 \%$, whereas it crystallized partially when $\phi_{\mathrm{PCL}} \geq 34 \%$ and the crystallinity increased with increasing $\phi_{\mathrm{PCL}}$, suggesting that $\phi_{\mathrm{PCL}}$ affects significantly the crystallizability of PCL blocks. However, crystallization was observed for all PCL- $b$-PS copolymers cast at $20^{\circ} \mathrm{C}$, and the crystallinity decreased appreciably with increasing the casting temperature. SAXS results revealed that high crystallinity PCL- $b$-PS copolymers had an intensity peak arising from the lamellar morphology, an alternating structure consisting of crystalline lamellae and amorphous layers, whereas low crystallinity PCL- $b$-PS copolymers did not show any SAXS peak, indicating the morphological difference among crystallized PCL- $b$-PS copolymers. The morphology formed in PCL- $b$-PS is discussed as a function of $\phi_{\mathrm{PCL}}$ in terms of the lamellar morphology observed for crystalline homopolymers.

KEY WORDS Crystalline-Amorphous Diblock Copolymer / Morphology / Crystallization / Glass

Transition /
\end{abstract}

The crystallization of block copolymers is very complicated since it starts from a homogeneous state or various microdomain structures existing in the melt. When the microdomain structure is weakly segregated, subsequent crystallization destroys this structure to result in a lamellar morphology, an alternating structure composed of crystalline lamellae and amorphous layers. ${ }^{1-10}$ This means that the driving force of crystallization is much larger than the stability of microdomain structures. The lamellar morphology in equilibrium has been experimentally investigated ${ }^{1,3,8}$ and also theoretically predicted, ${ }^{11,12}$ in which crystalline blocks are folded rather than extended to accommodate amorphous blocks between lamellae. When the molecular weight of block copolymers increases, the stability of microdomain structures increases and subsequent crystallization cannot destroy these structures, and eventually the constituent block crystallizes without a morphological reorganization. ${ }^{13-16}$

The glass transition of amorphous blocks is another factor to enhance the stability of microdomain structures. That is, the crystalline block cannot move freely when it crystallizes in the bulk since the amorphous block, linked with the crystalline block by the covalent bond, is anchored into glassy regions. Crystallization is, therefore, extremely restricted and may occur, for example, within the microdomain structure. In other words, these crystalline-amorphous diblock copolymers with a high glass transition temperature have potential to control the morphology formed by a combined effect of crystallization and microphase separation by using the vitrification of amorphous blocks.

There are experimental studies on the crystallization and final morphology formed in diblock and triblock copolymers consisting of crystalline and amorphous blocks, where the glass transition temperature of amorphous blocks is higher than the melting temperature of crystalline blocks. ${ }^{17-27}$ Gervais and Gallot first investigated the morphology of poly(ethylene oxide)-blockpolystyrene (PEO- $b$-PS) as a function of molecular characteristics of PEO- $b$-PS and crystallization conditions. ${ }^{17-20}$ Jerome and Teyssie extended the research to poly( $\varepsilon$-caprolactone)-block-PS (PCL- $b$-PS) copolymers, ${ }^{20-23}$ where they focussed their attention on morphology in a binary system of PCL- $b$-PS and diethyl phthalate, a good solvent only for PS blocks and discussed mainly the folding number of PCL blocks in equilibrium morphology. ${ }^{22}$ Later they investigated the crystallizability of PCL blocks in PCL- $b$-PS by calorimetric and optical methods as a function of copolymer composition and PCL block length. ${ }^{23}$ Liu et al. ${ }^{24,26,27}$ recently studied the morphology of poly(tetrahydrofuran)-block-poly(methyl methacrylate) (PTHF- $b$ PMMA) copolymers cast from the solution, and found that even in the microdomain structure PTHF crystallized partially though the crystallinity and melting temperature were strongly dependent on the copolymer composition (or type of microdomain structure).

In our earlier paper, ${ }^{28}$ we crystallized PCL- $b$-PS, where the glass transition temperature of the PS block, $T_{\mathrm{g}, \mathrm{PS}}$, was $c a .100^{\circ} \mathrm{C}$ and the melting temperature of the PCL block was $c a .60^{\circ} \mathrm{C}$. We added a plasticizer only to the PS block to lower $T_{\mathrm{g}, \mathrm{Ps}}$ and eventually induce the crystallization of PCL blocks. In this study, we crystallized PCL blocks in various PCL- $b$-PS copolymers by quenching from the melt and casting from the toluene

† To whom correspondence should be addressed (Phone: +81-761-51-1601, Fax: +81-761-51-1149, e-mail: nojima@jaist.ac.jp). 
solution. Final morphology was observed by small-angle $\mathrm{X}$-ray scattering (SAXS) and the melting behavior was investigated by differential scanning calorimetry (DSC). We elucidate the crystallizability of PCL blocks as a function of copolymer composition and discuss the resulting morphology observed by SAXS from the viewpoint of combined effects of crystallization, microphase separation, and glass transition.

\section{EXPERIMENTAL}

\section{Materials}

PCL- $b$-PS copolymers in this study were synthesized by a successive anionic polymerization under vacuum. The styrene monomer was first polymerized in toluene at room temperature for $24 \mathrm{~h}$ with $n$-buthyllithium as initiator, followed by the addition of an $\varepsilon$-caprolactone monomer to synthesize the block copolymer at $-15^{\circ} \mathrm{C}$ over 2-12 min. Reaction time and temperature of the $\varepsilon$-caprolactone monomer were carefully adjusted to prevent depolymerization (back-biting) by the living $\varepsilon$-caprolactone end during anionic growth of the PCL block. The styrene/initiator ratio and amount of $\varepsilon$ caprolactone monomers were changed to obtain various copolymers. The copolymer thus synthesized was precipitated several times into a large amount of methanol to remove the unreacted $\varepsilon$-caprolactone monomer completely.

The copolymers were characterized by gel permeation chromatography (GPC), vapor pressure osmometry (VPO), and nuclear magnetic resonance $\left({ }^{1} \mathrm{H}\right.$ NMR). Table I summarizes the results of molecular characterization, where the number after ' $\mathrm{CL}$ ' represents $\mathrm{PCL}$ vol\% $\left(=\phi_{\mathrm{PCL}}\right)$ in PCL- $b$-PS. $\phi_{\mathrm{PCL}}$ changes from 13 to $73 \%$, though the total molecular weight is not constant, which may seriously affect the stability of microdomain structures and also the size of the lamellar morphology as predicted by equilibrium theories for crystalline-amorphous block copolymers. ${ }^{1,12}$ The poly( $\varepsilon$-caprolactone) homopolymer, designated PCL1 in Table I, was also used to compare crystallization behavior and morphology with those of PCL- $b$-PS copolymers.

The glass transition temperature of the PS homopolymer is $c a .100^{\circ} \mathrm{C}$ and the melting temperature of the PCL homopolymer $T_{\mathrm{m} . \mathrm{PCL}}$ is $\mathrm{ca} .60^{\circ} \mathrm{C},{ }^{29}$ so that the crystallization of PCL blocks at temperatures below $T_{\mathrm{m}, \mathrm{PCL}}$ will be affected by the vitrification of PS blocks. PS and PCL homopolymers have no specific interactions between them and it is reported that the PCL homopolymer is partially miscible with the PS oligomer and not miscible with the PS homopolymer. ${ }^{29-31}$ This suggests that PCL- $b$-PS has a microdomain structure in the melt if the molecular weight is large enough.

\section{Sample Preparation}

Two methods were used to prepare the crystallized PCL- $b$-PS samples; quenching method and solutioncasting method. In the quenching method, PCL- $b$-PS was first annealed at $c a .110^{\circ} \mathrm{C}$ for $0.5 \mathrm{~h}$ to erase the previous thermal history and to develop the microdomain structure, then quenched into various crystallization temperatures $T_{\mathrm{c}}$ ranging from 20 to $50^{\circ} \mathrm{C}$, and crystallized for a long time. In the solution-casting method, toluene,
Table I. Characterization of polymers

\begin{tabular}{cccccc}
\hline Notation & Polymer & Source & Total $M_{n}{ }^{\mathrm{c}}$ & $M_{w} / M_{n}{ }^{\mathrm{d}}$ & $\begin{array}{c}\text { PCL:PS/ } \\
\text { vol } \%{ }^{\mathrm{e}}\end{array}$ \\
\hline CL13 & PCL- $b$-PS & a & 12000 & 1.18 & $13: 87$ \\
CL26 & PCL- $b$-PS & a & 23000 & 1.21 & $26: 74$ \\
CL34 & PCL- $b$-PS & a & 12000 & 1.41 & $34: 66$ \\
CL64 & PCL- $b$-PS & a & 30000 & 1.29 & $64: 36$ \\
CL73 & PCL- $b$-PS & a & 18000 & 1.75 & $73: 27$ \\
PCL1 & PCL & b & 7000 & 1.57 & - \\
\hline
\end{tabular}

${ }^{\text {a }}$ Synthesized in our laboratory. ${ }^{\mathrm{b}}$ Obtained from Scientific Polymer Products, Inc. ${ }^{\mathrm{c}}$ Determined by VPO. ${ }^{\mathrm{d}}$ Determined by GPC. ${ }^{\mathrm{e}}$ Determined by ${ }^{1} \mathrm{H}$ NMR.

good for both blocks, was used for the casting solvent and gradually evaporated at various $T_{\mathrm{c}}$ to obtain a crystallized thin film of PCL- $b$-PS. The complete evaporation of toluene was confirmed by no weight change on further evaporation.

\section{SAXS Measurements}

SAXS measurements were performed with a point focusing optics and a one-dimensional position sensitive proportional counter (PSPC) with an effective length of $10 \mathrm{~cm}$. SAXS optics have a curved monochrometer and curved mirror to focus the scattered intensity on the PSPC. $\mathrm{Cu}-\mathrm{K}_{\alpha}$ radiation by a MAC Science $\mathrm{M} 18 \mathrm{X}$ generator operating at $40 \mathrm{kV}$ and $30 \mathrm{~mA}$ was used throughout. The distance between the sample and PSPC was about $40 \mathrm{~cm}$. The geometry was further checked by a chicken tendon collagen, which gives a set of sharp diffractions corresponding to $65.3 \mathrm{~nm}$. The accumulation time for each measurement was $3000-5000$ s depending on the scattered intensity from the sample.

SAXS intensity was corrected for background scattering and absorption by the sample, and finally converted into the relative scattered intensity as a function of wave number $s$ defined as,

$$
s=(2 / \lambda) \sin \theta
$$

where $\lambda$ is the wave length of the incident $\mathrm{X}$-ray (= $0.1542 \mathrm{~nm}$ ) and $2 \theta$ is the scattering angle. The angular position of SAXS intensity peaks was evaluated after the SAXS curve was corrected for the Lorentz factor assuming that the morphology of crystallized PCL- $b$ PS copolymers is an alternating structure consisting of crystalline lamellae and amorphous layers (lamellar morphology).

The electron density difference between the amorphous PCL (337 e nm -3 at $100^{\circ} \mathrm{C}^{32}$ ) and amorphous PS (332 e $\mathrm{nm}^{-3}$ at $100^{\circ} \mathrm{C}^{33}$ ) is unfortunately extremely small, so we cannot expect the scattering from the microdomain structure in the melt. In fact, we observed a monotonously decreasing SAXS curve with increasing $s$ from molten CL13, CL26, and CL34. The scattering from the morphology after the crystallization of PCL blocks can be observed since there is enough electron density difference between PCL crystals $\left(393 \mathrm{e} \mathrm{nm}^{-3}\right.$ 34) and amorphous PS $\left(344 \mathrm{e} \mathrm{nm}^{-3}\right.$ at $\left.40^{\circ} \mathrm{C}^{33}\right)$.

\section{DSC Measurements}

A Perkin Elmer Model 7 DSC or Pyris 1 DSC was 

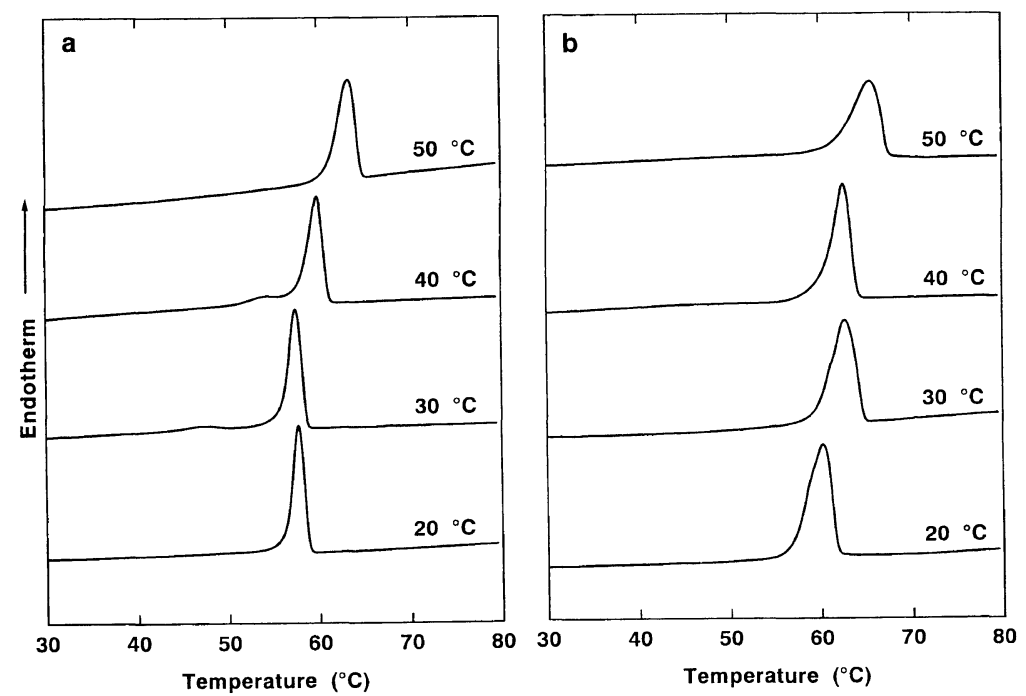

Figure 1. DSC thermograms of (a) CL73 quenched from the melt and (b) CL73 cast from the toluene solution. Crystallization temperature $T_{\mathrm{c}}$ is indicated on each curve. The heating rate is $5^{\circ} \mathrm{C} \mathrm{min}^{-1}$.

used to determine the melting temperature of PCL blocks $T_{\mathrm{m}, \mathrm{PCL}}$ and PCL crystallinity $\chi$ (i.e., $\mathrm{wt} \%$ of the crystallized PCL block against the existing PCL block in the system). The sample crystallized at each $T_{\mathrm{c}}$ for a long time was cooled quickly to $c a .20^{\circ} \mathrm{C}$ and then heated up to $120^{\circ} \mathrm{C}$ with a heating rate of $5^{\circ} \mathrm{Cmin}^{-1} . T_{\mathrm{m}, \mathrm{PCL}}$ was obtained from the endothermic peak position and $\chi$ was calculated as,

$$
\chi=\Delta H /\left(\Delta H^{0} \cdot f\right)
$$

where $\Delta H$ is the heat of fusion per gram of copolymers which can be evaluated from the peak area, $\Delta H^{0}$ that of perfect PCL crystals (135.44 $\mathrm{J} \mathrm{g}^{-132}$ ), and $f$ the weight fraction of PCL blocks.

\section{RESULTS AND DISCUSSION}

\section{DSC Measurements}

Figure 1 shows typical DSC curves measured for CL73 quenched from the melt (a) and cast from the toluene solution (b). Each DSC curve has an endothermic peak corresponding to the melting of PCL blocks and the peak position shifts slightly to the higher temperature with increasing crystallization temperature $T_{\mathrm{c}}$. The DSC curves measured for the quenched CL64 and CL34 have similar endothermic peaks shown in Figure 1a, but those for CL13 and CL26 do not have any peak for any $T_{\mathrm{c}}$ from 20 to $50^{\circ} \mathrm{C}$. This means that the PCL block does not crystallize at all by quenching when $\phi_{\mathrm{PCL}}$ is less than $26 \%$.

All solution-cast samples have an endothermic peak at $T_{\mathrm{c}}=20^{\circ} \mathrm{C}$. Crystallization is intuitively expected to be less restricted when PCL- $b$-PS is cast from the solution rather than quenched, and therefore the morphology may be different. The small endothermic peak observed at $T_{\mathrm{c}}=20^{\circ} \mathrm{C}$ for CL13 and CL26 disappears with increasing $T_{\mathrm{c}}$, which can be qualitatively explained by interplay between the crystallization and solvent-evaporation rates. The crystallization rate is usually smaller with increasing $T_{\mathrm{c}}$, which is exponentially retarded as a function of the degree of undercooling $\Delta T\left(=T_{\mathrm{m}}-T_{\mathrm{c}}\right){ }^{35}$ The crystallization of PCL blocks does not start or does
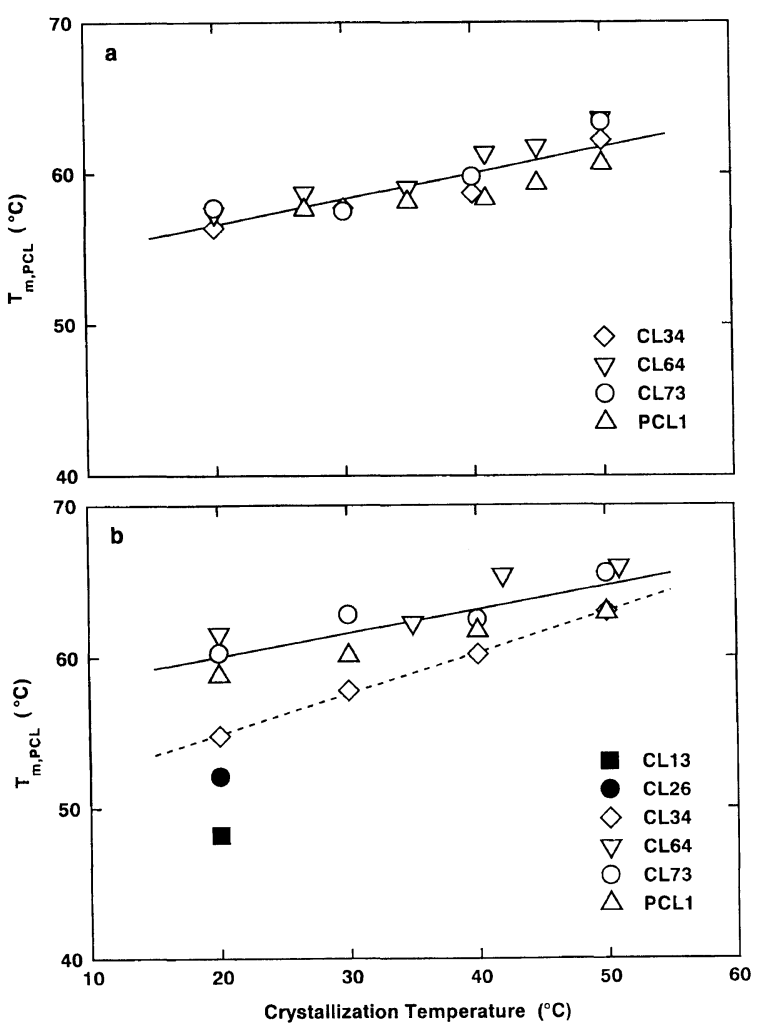

Figure 2. $T_{\mathrm{c}}$ dependence of melting temperature $T_{\mathrm{m}, \mathrm{PCL}}$ for (a) quenched samples and (b) solution-cast samples.

not end completely during the solvent-evaporation at a higher temperature or smaller $\Delta T$, the degree of which depends intimately on $\phi_{\mathrm{PCL}}$. Therefore, the solution-cast CL13 and CL26 do not crystallize at small $\Delta T$, and CL34, CL64, CL73, and PCL1 crystallize partially with immature crystallites formed in the system, yielding an appreciable decrease of $\chi$ with increasing $T_{\mathrm{c}}$. (Figure $3 \mathrm{~b}$ )

The melting temperature of the PCL block, $T_{\mathrm{m}, \mathrm{PCL}}$, is shown in Figure 2 as a function of $T_{\mathrm{c}}$ for all the samples quenched from the melt (a) and cast from the solution (b). $T_{\mathrm{m}, \mathrm{PCL}}$ for the quenched PCL- $b$-PS (with $\phi_{\mathrm{PCL}} \geq 34 \%$ ) makes a master curve irrespective of $\phi_{\mathrm{PCL}}$ and increases appreciably with increasing $T_{\mathrm{c}}$. It is well known that the 

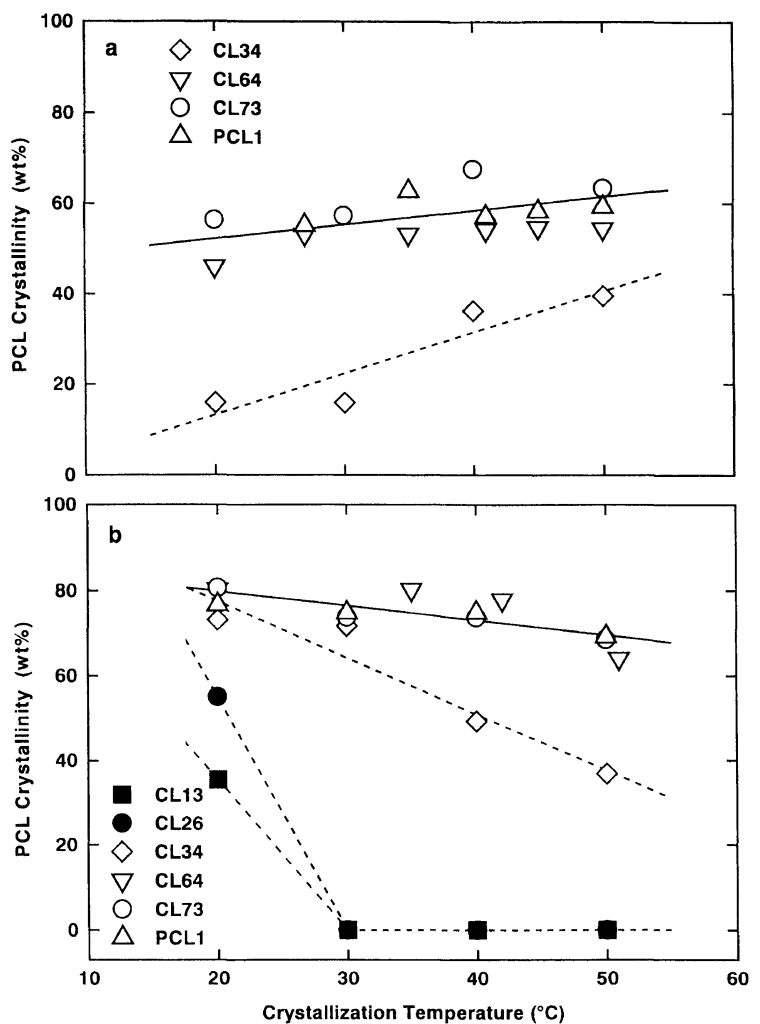

Figure 3. $T_{\mathrm{c}}$ dependence of PCL crystallinity $\chi$ (i.e., wt $\%$ of crystallized PCL blocks against total PCL blocks existing in the system) for (a) quenched samples and (b) solution-cast samples.

melting temperature of crystalline homopolymers, $T_{\mathrm{m}}$, is related to the lamellar thickness $l_{\mathrm{c}}$ as, ${ }^{36}$

$$
T_{\mathrm{m}}=T_{\mathrm{m}}^{0}\left\{1-2 \sigma /\left(l_{\mathrm{c}} \cdot \Delta H\right)\right\}
$$

where $T_{\mathrm{m}}^{0}$ is $T_{\mathrm{m}}$ of infinitely large crystals, $\sigma$ is the specific surface energy, and $\Delta H$ is the heat of fusion per unit volume. Therefore, Figure $2 \mathrm{a}$ means that $l_{\mathrm{c}}$ in the lamellar morphology is independent of $\phi_{\mathrm{PCL}}$ if $\phi_{\mathrm{PCL}} \geq 34 \%$ and $l_{\mathrm{c}}$ increases slightly with $T_{\mathrm{c}}$. $T_{\mathrm{m} \text {.PCL }}$ for the solution-cast PCL- $b$-PS is versatile, depending on $\phi_{\mathrm{PCL}}$ and $T_{\mathrm{c}} . T_{\mathrm{m} . \mathrm{PCL}}$ for PCL1, CL73, and CL64 again makes a straight line against $T_{\mathrm{c}}$ and is higher than that of the quenched samples at every $T_{\mathrm{c}} \cdot T_{\mathrm{m}, \mathrm{PCL}}$ decreases significantly with decreasing $\phi_{\mathrm{PCL}}$ at $T_{\mathrm{c}}=20^{\circ} \mathrm{C}$, while melting cannot be observed at $T_{\mathrm{c}} \geq 30^{\circ} \mathrm{C}$ for CL26 and CL13, as mentioned above.

We note in Figures $2 \mathrm{a}$ and $2 \mathrm{~b}$ that $T_{\mathrm{m}, \mathrm{PCL}}$ of CL64 and CL73 is generally higher than that of PCL1. In particular, $T_{\mathrm{m}, \text { PCL }}$ of the solution-cast CL73 at $T_{\mathrm{c}}=51^{\circ} \mathrm{C}$ is $66^{\circ} \mathrm{C}$, which is $\mathrm{ca} .4^{\circ} \mathrm{C}$ higher than that of PCL1. Since the equilibrium melting temperature of $\mathrm{PCL}$ is reported to be $70^{\circ} \mathrm{C},{ }^{37}$ the PCL block in CL73 is not fully extended but more extended than that in PCL1 if we assume that the difference in $T_{\mathrm{m}, \mathrm{PCL}}$ arises only from morphological effect. That is, the covalently linked PS block makes the PCL block more extended than PCL1 under the nonequilibrium crystallization. A kinetic effect is another plausible explanation. The molecular motion of PCL- $b$ PS is lower than that of PCL1 at around $T_{\mathrm{m}, \mathrm{PCL}}$ and therefore PCL- $b$-PS is less sensitive to heating measurements than PCL1 to yield a higher $T_{\mathrm{m}, \mathrm{PCL}}$. A careful study is necessary to understand the reasons for this.

Figure 3 shows plots of PCL crystallinity $\chi$, wt $\%$ of the crystallized PCL block against the total PCL block

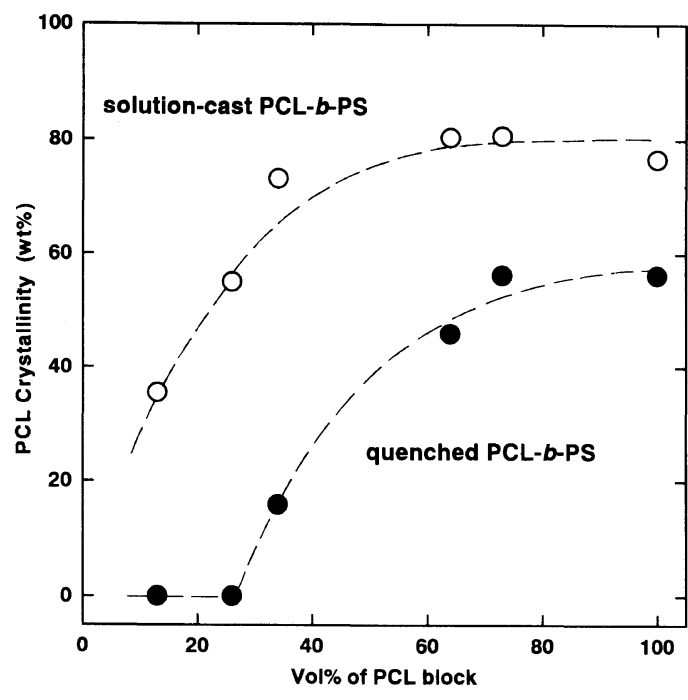

Figure 4. Composition (vol\% of PCL blocks in the copolymer) dependence of PCL crystallinity $\chi$ for quenched samples $(O)$ and solution-cast samples $(\bigcirc)$. Crystallization temperature is $20^{\circ} \mathrm{C}$.

in the system, against $T_{\mathrm{c}}$ for the quenched PCL-b-PS (a) and solution-cast PCL- $b$-PS (b). The $T_{\mathrm{c}}$ dependence of both samples is quite different; for the quenched PCL$b$-PS with $\phi_{\mathrm{PCL}} \geq 34 \%$ (Figure 3a), $\chi$ increases slightly with $T_{\mathrm{c}}$, as usually observed in crystalline homopolymers. This is qualitatively explained by the non-equilibrium crystallization at $T_{\mathrm{c}}$. The crystallization rate increases with decreasing $T_{\mathrm{c}}$ and immature (or thinner) lamellae are formed in the system to decrease $\chi$. Figure $3 \mathrm{a}$ also shows that $\chi$ depends intimately on $\phi_{\mathrm{PCL}}$, that is, $\chi$ decreases significantly at $\phi_{\mathrm{PCL}}=34 \%$ and eventually falls to zero with further decrease of $\phi_{\mathrm{PCL}}$. For the solutioncast PCL- $b$-PS (Figure $3 b) \chi$ decreases appreciably with increasing $T_{\mathrm{c}}$. In particular, $\chi$ for CL13 and CL26 changes dramatically with $T_{\mathrm{c}}$; when $T_{\mathrm{c}} \geq 30^{\circ} \mathrm{C}, \mathrm{CL} 13$ and CL26 cannot crystallize at all, whereas they crystallize partially at $T_{\mathrm{c}}=20^{\circ} \mathrm{C}$, as explained in Figure $2 \mathrm{~b}$.

Figure 4 shows plots of $\chi$ against $\phi_{\mathrm{PCL}}$ for the quenched PCL- $b$-PS $(\bullet)$ and solution-cast PCL- $b$-PS $(O)$. Heuschen et al. ${ }^{23}$ obtained a similar $\phi_{\mathrm{PCL}}$ dependence of $\chi$ for a series of PCL- $b$-PS copolymers with a constant PS block length $\left(M_{n} \sim 40000\right)$, and reached the conclusion that $\chi$ changed dramatically at $\phi_{\mathrm{PCL}}=40-45 \%$. Although the PCL block length is not constant in this study, $\chi$ changes smoothly with changing $\phi_{\mathrm{PCl}}$, suggesting that $\phi_{\mathrm{PCL}}$ affects seriously the crystallizability of PCL blocks rather than the PCL block length within the present molecular weight range. In particular, the quenched PCL- $b$-PS crystallizes partially when $\phi_{\mathrm{PCL}} \geq$ $34 \%$ though $\chi$ is significantly lower than that of the solution-cast PCL- $b$-PS. The crystallization of quenched PCL- $b$-PS indicates that the PCL block crystallizes within the existing microdomain structure or it crystallizes by accompanying a morphological reorganization from the microdomain structure into the lamellar morphology. The results of SAXS measurements reveal which morphology prevails in the quenched PCL- $b$-PS, and are presented in the next section.

\section{SAXS Measurements}

When crystalline homopolymers have lamellar mor- 

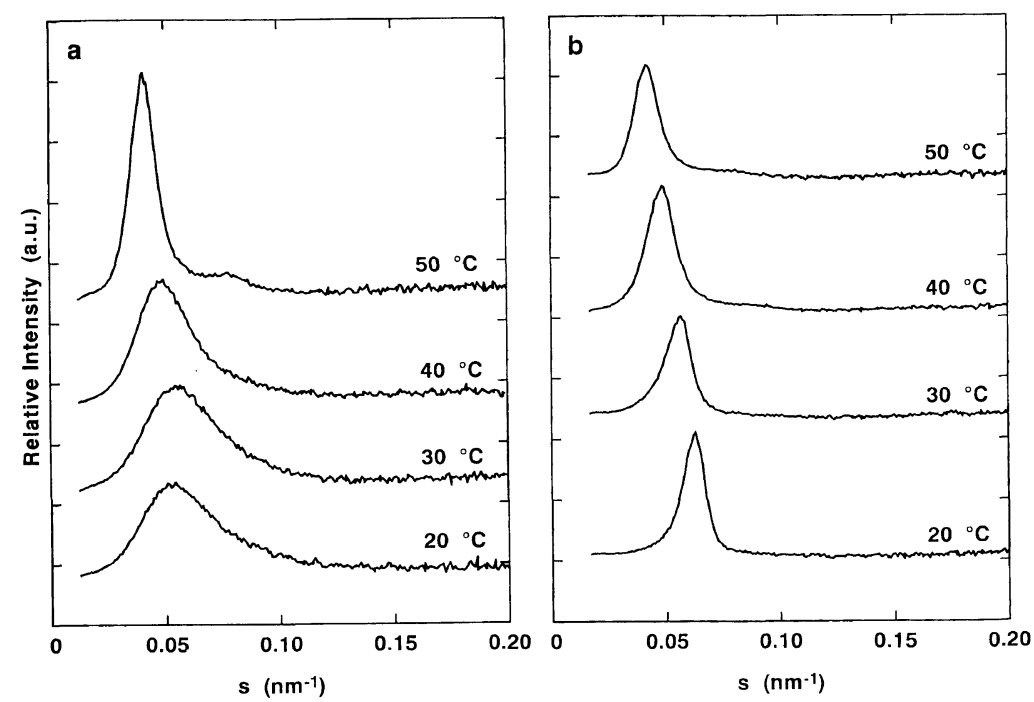

Figure 5. SAXS intensity curves of (a) CL73 quenched from the melt and (b) CL73 cast from the toluene solution. Crystallization temperature is indicated on each curve.

phology, the SAXS curve usually shows an intensity peak and we can evaluate the long spacing (or repeating distance) of this morphology from the angular position of the intensity peak. Figure 5 shows a typical example of SAXS curves for CL73 quenched from the melt (a) and cast from the solution (b) at each temperature indicated. In the case of PCL1, CL73, CL64, and solution-cast CL34, SAXS intensity peak is observed for all $T_{\mathrm{c}}$ investigated, and the angular position of the peak shifts significantly with changing $T_{\mathrm{c}}$. SAXS intensity peak for the quenched CL34, which shows an endothermic peak in the DSC measurement (Figure 2a), is not observed at every $T_{\mathrm{c}}$. That is, PCL blocks certainly crystallize in the system but crystallization does not yield the lamellar morphology detectable by SAXS, since there is a large PS block and this PS block prevents the formation of regular lamellar morphology.

When the morphology in the quenched sample is completely different from that in the solution-cast sample, the long spacing, which reflects morphology, differs significantly. It is also reported that crystallineamorphous block copolymers cast from the solution have lamellar morphology if the solvent is good for both blocks. The long spacing $L$ is plotted against $T_{\mathrm{c}}$ in Figure 6 for quenched (a) and solution-cast (b) samples, where $L$ of the quenched PCLl coincides substantially with that of the solution-cast PCL1. This suggests that lamellar morphology in PCL1 is not so dependent on the crystallization method (quenching or solution casting), though the lamellar thickness $l_{\mathrm{c}}$, amorphous layer thickness $l_{\mathrm{a}}$, and consequently crystallinity $\chi$ are altered slightly in the lamellar morphology, as described in Figures 2 and 3. This is true for CL64 and CL73 because $L$ of the quenched samples is almost the same with that of the solution-cast samples, indicating that the lamellar morphology is formed even in the quenched CL64 and CL73 probably due to the large amount of crystallizable block (PCL block). The difference in $L$ among PCL1, CL73, and CL64 can be quantitatively explained on the basis of lamellar morphology by considering PS blocks intervening between PCL lamellae, as described in the next section.
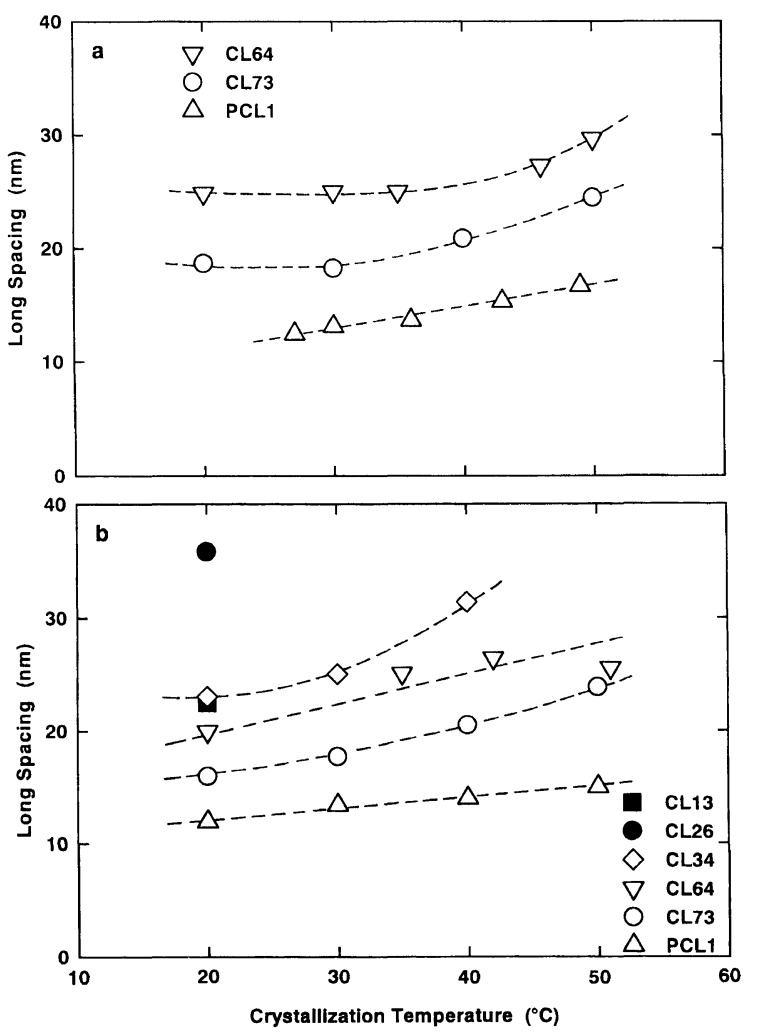

Figure 6. $\quad T_{\mathrm{c}}$ dependence of long spacing $L$ for (a) quenched samples and (b) solution-cast samples.

The SAXS intensity peak cannot be observed for the quenched CL34 though crystallization is confirmed by DSC. For the solution-cast CL34, the SAXS intensity peak is clearly observed. These results suggest that the morphology formed by two methods is different and the effect of glass transition is definitely recognized in the morphology formation of CL34. Unfortunately the type of microdomain structure in the molten CL34 cannot be estimated from the SAXS curve and PCL blocks should be surrounded by PS blocks judging from $\phi_{\mathrm{PCL}}$. Therefore, the PCL block is extremely restricted to crystallize in the quenched CL34 and eventually it crystallizes irregularly, for example, within the existing micro- 


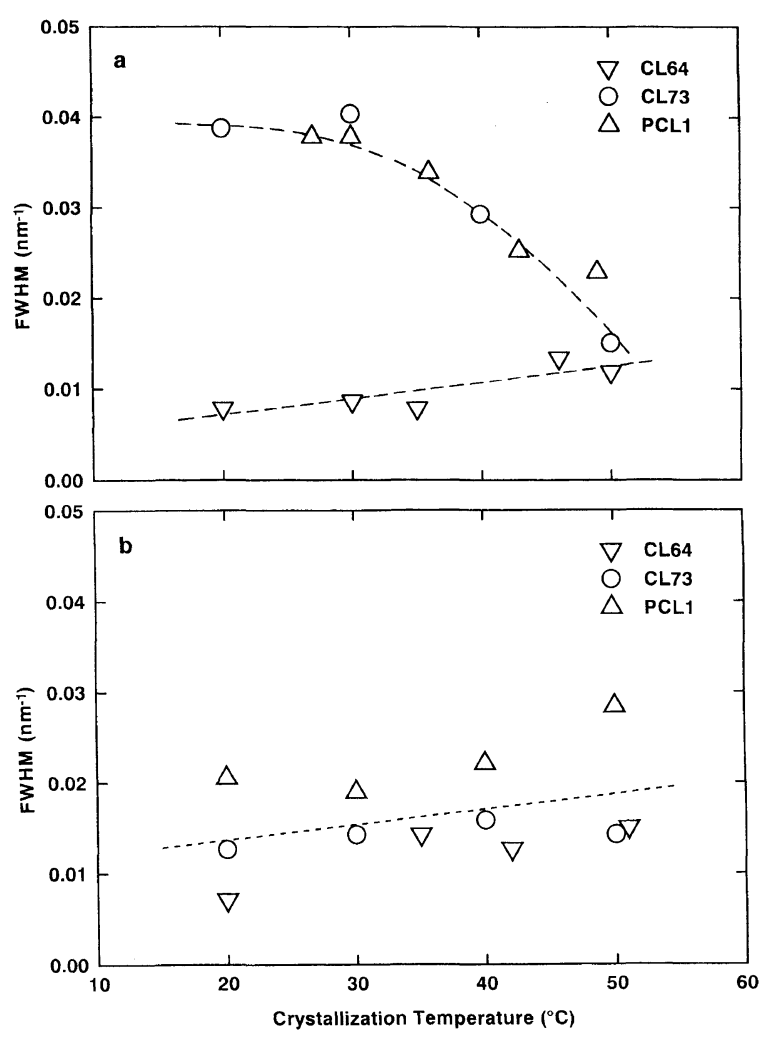

Figure 7. $T_{\mathrm{c}}$ dependence of FWHM for SAXS intensity peaks for (a) quenched samples and (b) solution-cast samples.

domain structure, leading to quite different morphology from that of the solution-cast CL34.

The large and irregular variation of $L$ for the solution-cast sample at $T_{\mathrm{c}}=20^{\circ} \mathrm{C}$ (Figure 6b) may be explained by taking the copolymer composition and total molecular weight into account. That is, the equilibrium lamellar morphology is determined by a subtle balance between stretching of PS blocks and chain-folding of PCL blocks, which depend intimately on the molecular characteristics of the copolymer, as theoretically predicted $^{11,12}$ and experimentally reported. ${ }^{8}$

Figure 7 shows the full width at half maximum (FWHM) of peak intensity for various samples indicated. Generally FWHM is a measure of the regularity of repeating structures. The quenched CL73 and PCL1 have large FWHM, which means the irregular lamellar morphology formed under the non-equilibrium crystallization. FWHM decreases with increasing $T_{\mathrm{c}}$. That is, the morphology becomes more regular at higher $T_{\mathrm{c}}$ because the crystallization rate decelerates with increasing $T_{\mathrm{c}}$ and morphology is slowly formed to approach to an equilibrium morphology formation with a narrow distribution of lamellar and amorphous layer thicknesses. FWHM for the quenched CL64 is extremely small and comparable to that of the solution-cast samples. (Figure 7b) The molecular weight of CL64 is highest among the copolymers used and the microdomain structure in the melt is comparatively stable. Thus, crystallization may start from an existing lamellar microdomain structure without further morphological reorganization, as in the case of an epitaxial crystallization recently reported in microphase separated block copolymers. ${ }^{38,39}$ It is necessary to investigate quantitatively the crystallization behavior and final morphology of a stable and definite

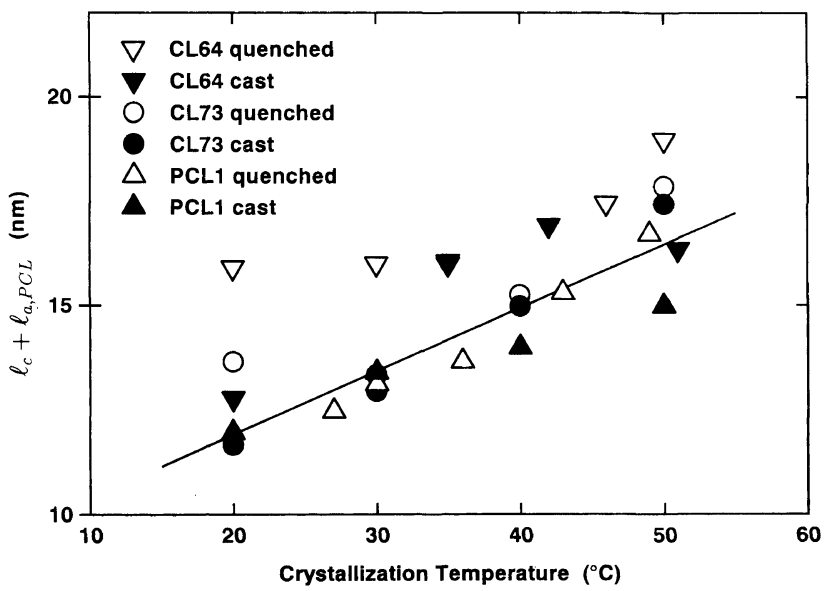

Figure 8. $T_{\mathrm{c}}$ dependence of $l_{\mathrm{c}}+l_{\mathrm{a}, \mathrm{PCL}}$ for quenched samples (open symbols) and solution-cast samples (closed symbols). The solid line represents least-squares of all data points except those of CL64.

microdomain structure confirmed by SAXS and TEM.

$T_{\mathrm{c}}$ dependence of FWHM for solution-cast samples is different from that for quenched samples; it increases rather than decreases with increasing $T_{\mathrm{c}}$. The interplay between crystallization and solvent-evaporation leads to accelerated non-equilibrium crystallization with increasing $T_{\mathrm{c}}$.

\section{Morphological Consideration}

It is necessary to confirm whether the SAXS intensity peak arises from the lamellar morphology or other morphologies, so that we consider the details of the morphology formed in PCL1, CL73, and CL64, since we have the SAXS intensity peak even for the quenched samples. It is well known that crystalline homopolymers form a lamellar morphology when quenched from the melt or cast from the solution, so that we assume here that SAXS intensity peak for PCL1, irrespective of the crystallization method and $T_{\mathrm{c}}$, arises from lamellar morphology. Also, it is plausible to assume that the PS block is segregated completely from the amorphous PCL block in the lamellar morphology with a sharp interface between them because PCL is not miscible with PS. ${ }^{29-31}$ This leads to the equation,

$$
l_{\mathrm{c}}+l_{\mathrm{a}, \mathrm{PCL}}=\left(\phi_{\mathrm{PCL}}^{\prime} / 100\right) \times L
$$

where $l_{\mathrm{a}, \mathrm{PCL}}$ is the amorphous PCL layer thickness and $\phi_{\mathrm{PCL}}^{\prime}$ is $\mathrm{vol} \%$ of the crystallized and amorphous PCL blocks in the system and almost equals to $\phi_{\mathrm{PCL}}$. We extract the PCL region in the lamellar morphology after excluding the PS block with different sizes by eq 4 considering the $T_{\mathrm{c}}$ dependence of the morphology.

Figure 8 shows plots of $l_{\mathrm{c}}+l_{\mathrm{a}, \text { PCL }}$ as a function of $T_{\mathrm{c}}$ for PCL1, CL73, and CL64 quenched from the melt (open symbols) and cast from the solution (closed symbols). All the data points fall on a same curve irrespective of copolymers and crystallization methods though data for CL64 are systematically large probably due to the effects of stable microdomain structures. Figure 8 suggests that the alternating structure of crystalline and amorphous PCL blocks with similar spacings is certainly formed in CL73 and CL64 as well as PCL1, and the PS block is accommodated within the amorphous layer between PCL lamellae. 


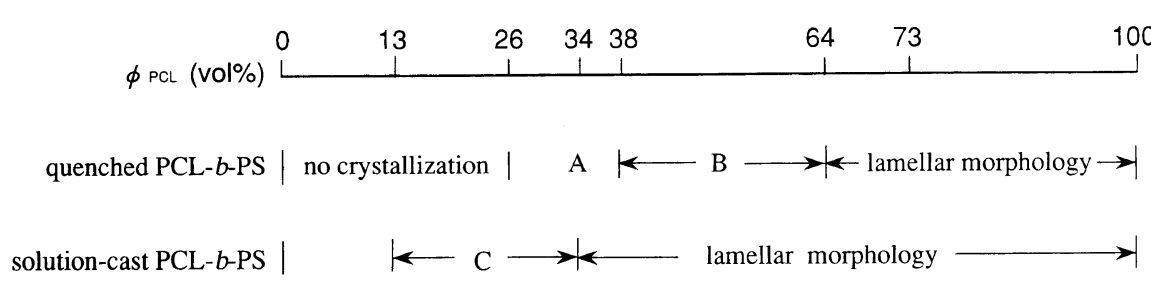

Figure 9. Summary of morphology in PCL- $b$-PS as a function of $\phi_{\mathrm{PCL}}$. A, crystallization detected by DSC but not SAXS; B, lamellar morphology confirmed by Herman et al. ${ }^{22} ; \mathrm{C}$, crystallization observed at $T_{\mathrm{c}}=20^{\circ} \mathrm{C}$ but not $T_{\mathrm{c}} \geqq 30^{\circ} \mathrm{C}$.

The morphological speculation of CL34 is quite difficult only from the present results because the morphology is different for quenched and solution-cast copolymers and also SAXS results do not provide useful information on the morphology of quenched CL34. But the morphology formed in CL34 is interesting, since we can control it by the crystallization method. It is, therefore, necessary to understand the crystallization mechanism and final morphology of CL34 precisely with other methods.

The morphology in PCL- $b$-PS obtained in this study, together with the result reported by Herman et al., ${ }^{22}$ is summarized in Figure 9 as a function of $\phi_{\mathrm{PCL}}$. Thus, $\phi_{\mathrm{PCL}}$ has significantly affected the crystallization of PCL blocks and final morphology in the present molecular weight range, in particular, when PCL- $b$-PS is quenched from the melt.

\section{CONCLUSIONS}

In this study, we crystallized PCL- $b$-PS copolymers by quenching and solution-casting methods, where the crystallization of PCL blocks was restricted by vitrification of PS blocks. The following conclusions were obtained.

1. We observed the crystallization of PCL blocks in quenched PCL- $b$-PS copolymers when $\phi_{\mathrm{PCL}}$ was larger than $64 \%$ (CL64 and CL73) both by DSC and SAXS measurements. The long spacing $L$ agreed with that of PCL- $b$-PS cast from the toluene solution, indicating lamellar morphology even in the quenched PCL- $b$-PS. The increase of $L$ for CL73 and CL64 was quantitatively explained on the basis of the lamellar morphology with the additional PS block intervening between PCL lamellae.

2. When $\phi_{\text {PCL }}$ was $34 \%$ (CL34), we observed the crystallization of PCL blocks in the quenched PCL- $b$-PS by DSC but the SAXS intensity peak could not be observed, suggesting the PCL block crystallized partially in CL34 and the regular morphology detectable by SAXS did not form. However, lamellar morphology was confirmed for the solution-cast CL34.

3. When $\phi_{\mathrm{PCL}}$ was less than $26 \%$ (CL26 and CL13), no crystallization was observed for quenched samples, but partial crystallization was detected for solution-cast samples at $T_{\mathrm{c}}=20^{\circ} \mathrm{C}$. Since the PCL block is surrounded by many PS blocks in the melt, crystallization is extremely restricted when quenched from the melt, resulting in no crystallization.

Acknowledgments. This work was supported in part by Tokuyama Science Foundation and by Grants-in-Aid for Scientific Research (No. 09650992) from the Ministry of Education, Science, Sports and Culture of Japan.

\section{REFERENCES}

1. R. Unger, D. Beyer, and E. Donth, Polymer, 32, 3305 (1991).

2. S. Nojima, K. Kato, S. Yamamoto, and T. Ashida, Macromolecules, 25, 2237 (1992).

3. P. Rangarajan, R. A. Register, and L. J. Fetters, Macromolecules, 26, 4640 (1993).

4. S. Nojima, H. Nakano, and T. Ashida, Polym. Commun., 34, 4168 (1993).

5. S. Nojima, H. Nakano, Y. Takahashi, and T. Ashida, Polymer, 35, 3479 (1994).

6. P. Rangarajan, R. A. Register, D. H. Adamson, L. J. Fetters, W. Bras, S. Naylor, and A. J. Ryan, Macromolecules, 28, 1422 (1995)

7. A. J. Ryan, I. W. Hamley, W. Bras, and F. S. Bates, Macromolecules, 28, 3860 (1995).

8. S. Nojima, S. Yamamoto, and T. Ashida, Polym. J., 27, 673 (1995).

9. Z. Gan, B. Jiang, and J. Zhang, J. Appl. Polym. Sci., 59, 961 (1996).

10. S. M. Mai, J. P. A. Fairclough, K. Viras, P. A. Gorry, I. W. Hamley, A. J. Ryan, and C. Booth, Macromolecules, 30, 8392 (1997).

11. E. A. DiMarzio, C. M. Guttman, and J. D. Hoffman, Macromolecules, 13, 1194 (1980).

12. M. D. Whitmore and J. Noolandi, Macromolecules, 21, 1482 (1988).

13. K. C. Douzinas and R. E. Cohen, Macromolecules, 25, 5030 (1992).

14. I. W. Hamley, J. P. A. Fairclough, A. J. Ryan, F. S. Bates, and E. T. Andrews, Polym. Commun., 37, 4425 (1996).

15. D. J. Quiram, R. A. Register, and G. R. Marchand, Macromolecules, 30, 4551 (1997).

16. S. Nojima, K. Hashizume, A. Rohadi, and S. Sasaki, Polymer, 38, 2711 (1997).

17. M. Gervais and B. Gallot, Makromol. Chem., 178, 1577 (1977).

18. M. Gervais and B. Gallot, Makromol. Chem., 171, 157 (1973).

19. M. Gervais and B. Gallot, Polymer, 22, 1129 (1981).

20. M. Gervais, B. Gallot, R. Jerome, and P. Teyssie, Makromol. Chem., 182, 989 (1981).

21. J. J. Herman, R. Jerome, P. Teyssie, M. Gervais, and B. Gallot, Makromol. Chem., 179, 1111 (1978).

22. J. J. Herman, R. Jerome, P. Teyssie, M. Gervais, and B. Gallot, Makromol. Chem., 182, 997 (1981).

23. J. Heuschen, R. Jerome, and P. Teyssie, J. Polym. Sci., B27, 523 (1989).

24. L. Liu, H. Li, B. Jiang, and E. Zhou, Polymer, 35, 5511 (1994).

25. V. Balsamo, F. Gyldenfeldt, and R. Stadler, Macromol. Chem. Phys., 197, 3317 (1996).

26. L. Liu, B. Jiang, and E. Zhou, Polymer, 37, 3937 (1996).

27. L. Liu, F. Yeh, and B. Chu, Macromolecules, 29, 5336 (1996).

28. S. Nojima, H. Tanaka, A. Rohadi, and S. Sasaki, Polymer, 39, 1727 (1998).

29. S. Nojima, Y. Terashima, and T. Ashida, Polymer, 27, 1007 (1986).

30. H. Tanaka and T. Nishi, Phys. Rev. A, 39, 783 (1989).

31. S. Nojima, K. Satoh, and T. Ashida, Macromolecules, 24, 942 (1991).

32. V. Crescenzi, G. Manzini, G. Galzolari, and C. Borri, Eur. Polym. $J ., 8,449$ (1972).

33. M. J. Richardson and N. G. Savil, Polymer, 18, 3 (1977)

34. K. Ito and Y. Yamashita, Macromolecules, 11, 68 (1978). 
35. L. Mandelkern, "Crystallization of Polymers," McGraw-Hill, New York, N.Y., 1964.

36. B. Wunderlich, "Macromolecular Physics 3," Academic Press, New York, N.Y., 1980.

37. P. J. Phillips, G. J. Rensch, and K. D. Taylor, J. Polym. Sci., B25, 1725 (1987).
38. I. W. Hamley, J. P. A. Fairclough, N. J. Terrill, A. J. Ryan, P. M. Lipic, F. S. Bates, and E. T. Andrews, Macromolecules, 29 , 8835 (1996)

39. A. J. Ryan, J. P. A. Fairclough, I. W. Hamley, S. M. Mai, and C. Booth, Macromolecules, 30, 1723 (1997). 